\title{
Commuting Separately Quasihomogeneous Small Hankel Operators on Pluriharmonic Bergman Space
}

\author{
Qi Wu, ${ }^{1}$ Chuntao Qin, ${ }^{1}$ Yong Chen $\mathbb{D}^{1},{ }^{1}$ and Yile Zhao ${ }^{2}$ \\ ${ }^{1}$ Department of Mathematics, Zhejiang Normal University, Jinhua 321004, China \\ ${ }^{2}$ Department of Mathematics, Hangzhou Normal University, Hangzhou, Zhejiang 310036, China
}

Correspondence should be addressed to Yong Chen; ychen227@gmail.com

Received 28 November 2017; Revised 17 January 2018; Accepted 28 January 2018; Published 1 March 2018

Academic Editor: Nikolai L. Vasilevski

Copyright (c) 2018 Qi Wu et al. This is an open access article distributed under the Creative Commons Attribution License, which permits unrestricted use, distribution, and reproduction in any medium, provided the original work is properly cited.

We study (semi)commutativity of small Hankel operators with separately quasihomogeneous symbols on the pluriharmonic Bergman space of the unit ball. Some product problems are also concerned.

\section{Introduction}

Let $\mathbb{B}_{n}$ be the unit ball in $\mathbb{C}^{n}$ and its boundary $\mathbb{S}_{n}$. Let $d v$ denote the normalized Lebesgue volume measure on the unit ball $\mathbb{B}_{n} . L^{2}\left(\mathbb{B}_{n}, d v\right)$ is the Hilbert space of Lebesgue square integrable functions on $\mathbb{B}_{n}$ with inner product:

$$
\langle f, g\rangle=\int_{\mathbb{B}_{n}} f(z) \overline{g(z)} d v(z) .
$$

The Bergman space $L_{a}^{2}\left(\mathbb{B}_{n}\right)$ is a subspace of $L^{2}\left(\mathbb{B}_{n}, d v\right)$ consisting of all holomorphic functions. It is well known that $L_{a}^{2}\left(\mathbb{B}_{n}\right)$ is a reproducing function space with the reproducing kernel:

$$
K_{z}(w)=\frac{1}{(1-\langle w, z\rangle)^{n+1}}
$$

for $z, w \in \mathbb{B}_{n}$. Let $P$ be the orthogonal projection from $L^{2}\left(\mathbb{B}_{n}, d v\right)$ onto $L_{a}^{2}\left(\mathbb{B}_{n}\right)$; then we have

$$
P f(z)=\left\langle f, K_{z}\right\rangle, \quad f \in L^{2}\left(\mathbb{B}_{n}, d v\right) .
$$

The pluriharmonic Bergman space $L_{h}^{2}\left(\mathbb{B}_{n}\right)$ is the closed subspace of $L^{2}\left(\mathbb{B}_{n}, d v\right)$ consisting of the pluriharmonic functions on $\mathbb{B}_{n}$. It is well known that

$$
L_{h}^{2}\left(\mathbb{B}_{n}\right)=L_{a}^{2}\left(\mathbb{B}_{n}\right)+\overline{L_{a}^{2}\left(\mathbb{B}_{n}\right)},
$$

where $\overline{L_{a}^{2}\left(\mathbb{B}_{n}\right)}=\left\{\bar{f} \mid f \in L_{a}^{2}\left(\mathbb{B}_{n}\right)\right\}$; then $L_{h}^{2}\left(\mathbb{B}_{n}\right)$ is also a reproducing function space with reproducing kernel:

$$
R_{z}(w)=K_{z}(w)+\overline{K_{z}(w)}-1, \quad z, w \in \mathbb{B}_{n} .
$$

Let $Q$ denote the orthogonal projection from $L^{2}\left(\mathbb{B}_{n}, d v\right)$ onto $L_{h}^{2}\left(\mathbb{B}_{n}\right)$; then

$$
Q f(z)=\left\langle f, R_{z}\right\rangle, \quad f \in L^{2}\left(\mathbb{B}_{n}, d v\right) .
$$

Using (5), we have

$$
Q f(z)=P f(z)+\overline{P \bar{f}(z)}-P f(0) .
$$

Let $L^{\infty}$ be a set of all bounded measurable functions on $\mathbb{B}_{n}$. Fix a function $\varphi \in L^{\infty}$. The Toeplitz operator $T_{\varphi}$ : $L_{h}^{2}\left(\mathbb{B}_{n}\right) \rightarrow L_{h}^{2}\left(\mathbb{B}_{n}\right)$ and the small Hankel operator $H_{\varphi}:$ $L_{h}^{2}\left(\mathbb{B}_{n}\right) \rightarrow L_{h}^{2}\left(\mathbb{B}_{n}\right)$ with the symbol $\varphi$ are defined by

$$
\begin{gathered}
T_{\varphi}(f)=Q(\varphi f), \\
H_{\varphi}(f)=Q J(\varphi f),
\end{gathered}
$$

respectively, where $J: L^{2}\left(\mathbb{B}_{n}, d v\right) \rightarrow L^{2}\left(\mathbb{B}_{n}, d v\right)$ is an unitary operator defined by $J f(z)=f(\bar{z})$. One can verify that $J Q=$ $Q J$, so we see the relation between the Toeplitz operator and small Hankel operator is

$$
H_{\varphi}=J T_{\varphi}=T_{J \varphi} J .
$$


One can easily check $T_{\varphi}^{*}=T_{\bar{\varphi}}$, so, by the above relation, we have $H_{\varphi}^{*}=H_{\varphi^{*}}$, and here $\varphi^{*}(z)=\overline{\varphi(\bar{z})}$.

We need more notations. Let $\mathbb{N}$ denote the set of all nonnegative integers. For multi-index $\alpha=\left(\alpha_{1}, \alpha_{2}, \ldots, \alpha_{n}\right) \in$ $\mathbb{N}^{n}$ and point $z=\left(z_{1}, z_{2}, \ldots, z_{n}\right) \in \mathbb{B}_{n}$, we write

$$
\begin{aligned}
& |\alpha|=\alpha_{1}+\alpha_{2}+\cdots+\alpha_{n}, \\
& \alpha !=\alpha_{1} ! \cdots \alpha_{n} ! \\
& z^{\alpha}=z_{1}^{\alpha_{1}} \cdots z_{n}^{\alpha_{n}} .
\end{aligned}
$$

For two multi-indexes $\alpha=\left(\alpha_{1}, \alpha_{2}, \ldots, \alpha_{n}\right), \beta=\left(\beta_{1}, \beta_{2}, \ldots\right.$, $\left.\beta_{n}\right) \in \mathbb{N}^{n}$, the notations $\alpha \geq \beta$ means that $\alpha_{j} \geq \beta_{j}$ for every $j$ and $\alpha \perp \beta$ means that

$$
\alpha_{1} \beta_{1}+\alpha_{2} \beta_{2}+\cdots+\alpha_{n} \beta_{n}=0
$$

Let $\alpha-\beta$ denote $\left(\alpha_{1}-\beta_{1}, \alpha_{2}-\beta_{2}, \ldots, \alpha_{n}-\beta_{n}\right)$. Note that if $\alpha-\beta \geq 0=(0,0, \ldots, 0)$, then $|\alpha-\beta|=|\alpha|-|\beta|$.

For a function $\phi \in L^{2}\left(\mathbb{B}_{n}, d v\right), \phi$ is said to be radial if $\phi(z)=\phi(|z|)$ and separately radial if $\phi\left(z_{1}, z_{2}, \ldots, z_{n}\right)=$ $\phi\left(\left|z_{1}\right|,\left|z_{2}\right|, \ldots,\left|z_{n}\right|\right)$.

Let $r=\left(r_{1}, r_{2}, \ldots, r_{n}\right),|r|=\sqrt{\left|r_{1}\right|^{2}+\left|r_{2}\right|^{2}+\cdots+\left|r_{n}\right|^{2}}$. For $f \in L^{2}\left(\mathbb{B}_{n}, d v\right)$, if there are $\beta, \gamma \in \mathbb{N}^{n}$ and separately radial function $\phi$ such that $f(|r| \xi)=\xi^{\beta} \bar{\xi}^{\gamma} \phi(r)$, where $\xi \in \mathbb{S}_{n}$, then $f$ is called a separately quasihomogeneous function.

The (semi)commutativity of two operators is an important topic in operator theory. In [1], Brown and Halmos completely characterized the commutativity of Toeplitz operators on the classical Hardy space. From then on, many related works on Toeplitz operators or (small) Hankel operators emerged (see, e.g., $[2,3]$ ).

For the case on the Bergman space of the unit disk, the commutativity is more subtle than that on the Hardy space. References [4,5] obtained the Brown-Halmos type theorems for Toeplitz operators with harmonic symbols. Many subsequent works studied these problems for special symbol classes, such as harmonic symbols, radial symbols, or quasihomogeneous symbols; see [6-9], for example.

On the harmonic Bergman space, there were some studies focusing on the commuting Toeplitz operators with harmonic symbols $[10,11]$ or quasihomogeneous symbols $[12,13]$ and showed that the results obtained are also quite different from the case on the Hardy or Bergman space. Recently, [14] studied the algebraic properties of small Hankel operators on the harmonic Bergman space and got very different commutativity of small Hankel operators compared with the case of Toeplitz operators. So in this paper, we want to continue the work and generalize the commuting small Hankel operators to the higher dimension case.

In order to state our main results, we still need the following notations and facts. Denote $\tau\left(\mathbb{B}_{n}\right)$ as the set

$$
\left\{r=\left(r_{1}, \ldots, r_{n}\right)=\left(\left|z_{1}\right|, \ldots,\left|z_{n}\right|\right):\left(z_{1}, \ldots, z_{n}\right) \in \mathbb{B}_{n}\right\} .
$$

If $\phi$ is a bounded separately radial function, then

$$
\int_{\mathbb{B}_{n}} \phi(z) d v(z)=2^{n} n ! \int_{\tau\left(\mathbb{B}_{n}\right)} \phi(r) r d r
$$

where $r d r=\prod_{i=1}^{n} r_{i} d r_{i}$. Let $\mathscr{R}$ be the set consisting of all $\phi \epsilon$ $L^{2}(\mathbb{B}, d v)$ which is separately radial. Note that for every $h \in$ $L^{2}\left(\mathbb{B}_{n}, d v\right)$ we have

$$
h(|r| \xi)=\sum_{\beta, \gamma \geq 0} \xi^{\beta} \bar{\xi}^{\gamma} \phi_{\beta, \gamma}(r),
$$

where every $\phi_{\beta, \gamma} \in \mathscr{R}$. Moreover, if $h \in L^{\infty}$, then also each $\xi^{\beta} \bar{\xi}^{\gamma} \phi_{\beta, \gamma} \in L^{\infty}$. For details, one may see [15].

We will first investigate when two small Hankel operators with a certain symbols commute. For two operators $T_{1}, T_{2}$, their commutator $T_{1} T_{2}-T_{2} T_{1}$ is denoted by $\left[T_{1}, T_{2}\right]$. So $T_{1}$ commutes with $T_{2}$ means $\left[T_{1}, T_{2}\right]=0$.

Theorem 1. Let $p, q, s, t \in \mathbb{N}^{n}$ and $\phi, \psi \in \mathscr{R} \cap L^{\infty}$. Then $\left[H_{\xi^{p} \bar{\xi}^{s} \phi}, H_{\xi^{q} \bar{\xi}^{t} \psi}\right]=0$ if and only if one of the following holds:

(i) $\phi=0$ or $\psi=0$.

(ii) $p-s=q-t$.

Note that $H_{\xi^{p} \bar{\xi}_{\phi}^{s}}^{*}=H_{\xi^{p} \bar{\xi}^{s} \bar{\phi}}$, so the above theorem implies that $H_{\xi p \bar{\xi} \phi}$ is always a normal operator on $L_{h}^{2}\left(\mathbb{B}_{n}\right)$. We also note that it is still open when two Toeplitz operators with separately quasihomogeneous symbols commute.

Theorem 2. Let $\phi$ be bounded radial and $h \in L^{\infty}$. Then $\left[H_{\phi}, H_{h}\right]=0$ if and only if one of the following holds:

(i) $\phi=0$.

(ii) $h$ is separately radial.

(iii) $\phi$ is nonzero constant and $J h=h$.

The above result is also different from the case for two Toeplitz operators with same symbols; see Corollary 5.2 in [15].

In order to get the semicommutativity of small Hankel operators (see Corollaries 12 and 15 in the next section), we turn to characterize when the product of two small Hankel operators is another small Hankel operator. We obtain the following results.

Theorem 3. Let $p, q, s, t \in \mathbb{N}^{n}$ and $\phi, \psi \in \mathscr{R} \cap L^{\infty}, h \in L^{\infty}$. Then the following statements are equivalent:

(i) $H_{\xi^{p} \bar{\xi}_{\phi}^{s}} H_{\xi^{q} \bar{\xi}_{\psi}^{t}}=H_{h}$.

(ii) $H_{\xi^{q} \bar{\xi}^{t} \psi} H_{\xi p \bar{\xi}^{s} \phi}=H_{h}$.

(iii) $\phi=h=0$ or $\psi=h=0$.

Theorem 4. Let $\phi$ be bounded radial and $g, h \in L^{\infty}$. Then the following statements are equivalent:

(i) $H_{\phi} H_{h}=H_{g}$.

(ii) $H_{h} H_{\phi}=H_{g}$.

(iii) $\phi=g=0$ or $h=g=0$. 
We would like to point out that the above main results also can apply to answer other related questions. For an example in point, by (9), we have

$$
T_{g} T_{J h}-T_{h} T_{J g}=J\left(H_{g} H_{h}-H_{h} H_{g}\right) J
$$

so one obtains that when $\left[H_{g}, H_{h}\right]=0$ can also answer when $T_{g} T_{J h}=T_{h} T_{J g}$.

We will give the proofs of above main results in the next section. Meanwhile, several corollaries also will be deduced.

\section{Proof of the Main Results}

We first recall some known facts.

It is well known that a bounded analytic function on the half plane $\{z \in \mathbb{C}: \operatorname{Re} z>0\}$ is uniquely determined by its value on an arithmetic sequence of integers. In fact, we have the following classical theorem (see p. 102 of [16]).

Lemma 5. Suppose that $f$ is a bounded analytic function on $\{z \in \mathbb{C}: \operatorname{Re} z>0\}$ which vanishes at the pairwise distinct points $z_{1}, z_{2}, \ldots$, where $\inf \left\{\left|z_{j}\right|\right\}>0$ and $\sum_{j \geq 1} \operatorname{Re}\left(1 / z_{j}\right)=\infty$, and then $f$ vanishes identically on $\{z \in \mathbb{C}: \operatorname{Re} z>0\}$.

We will need a similar result in higher dimensions which is proved by the above lemma. First, we give the following notations.

Let $E \subset \mathbb{N}^{2}$, we say that $E$ satisfies condition (I) if there exists a sequence $\left\{\alpha_{i}^{1}\right\}_{i=1}^{\infty}$ such that $\sum_{i=1}^{\infty}=1 / \alpha_{i}^{1}=\infty$, and, for every fixed $\alpha_{i}^{1}$, there also exists a sequence $\left\{\alpha_{i j}^{2}\right\}_{j=1}^{\infty}$ such that $\sum_{j=1}^{\infty}\left(1 / \alpha_{i j}^{2}\right)=\infty$ and $\left\{\left(\alpha_{i}^{1}, \alpha_{i j}^{2}\right): j=1,2, \ldots\right\} \subset E$.

One will easily see that for a multi-index $\delta \in \mathbb{N}^{2}$, if $E \subset\left\{\alpha \in \mathbb{N}^{2}: \alpha \geq \delta\right\}$ and let $E^{c}$ be the complement of $E$ in $\left\{\alpha \in \mathbb{N}^{2}: \alpha \geq \delta\right\}$, then either $E$ or $E^{c}$ satisfies the condition (I). Using Lemma 5, one may prove the following (also see Corollary 2.7 in [15]).

Lemma 6. Let $p, s \in \mathbb{N}^{2}$ and $g(r)$ be a bounded function on $\tau\left(\mathbb{B}_{2}\right)$. If the set

$$
E=\left\{\alpha \in \mathbb{N}^{2}: \alpha \geq s, \int_{\tau\left(\mathbb{B}_{2}\right)} g(r) r^{2 \alpha+p-s} r d r=0\right\}
$$

satisfies condition (I), then $g=0$.

Let $\alpha, p, s \in \mathbb{N}^{n}$. Set

$$
A(\phi ; \alpha, p, s)
$$

$$
=: \frac{2^{n}(n+|\alpha+p-s|) ! \int_{\tau\left(\mathbb{B}_{n}\right)} \phi(r) r^{2 \alpha+2 p}|r|^{-|p|-|s|} r d r}{(\alpha+p-s) !},
$$

when $\alpha+p \geq s$ and

$$
\begin{aligned}
& B(\phi ; s, \alpha, p) \\
& =: \frac{2^{n}(n+|s-\alpha-p|) ! \int_{\mathcal{\tau}\left(\mathbb{B}_{n}\right)} \phi(r) r^{2 s}|r|^{-|p|-|s|} r d r}{(s-\alpha-p) !}
\end{aligned}
$$

when $\alpha+p \leq s$. The following lemma is immediately from (9) and Lemma 3.4 of [17] and we will use it frequently.
Lemma 7. Let $p, s \in \mathbb{N}^{n}$ and $\phi \in \mathscr{R}$; then, for any multi-index $\alpha \in \mathbb{N}^{n}$

$$
\begin{aligned}
& H_{\xi p \bar{\xi}^{s} \phi}\left(z^{\alpha}\right)= \begin{cases}A(\phi ; \alpha, p, s) \bar{z}^{\alpha+p-s} & \alpha+p \geq s, \\
B(\phi ; s, \alpha, p) z^{s-\alpha-p} & \alpha+p \leq s, \\
0 & \text { others, }\end{cases} \\
& H_{\xi^{p} \bar{\xi}^{s} \phi}\left(\bar{z}^{\alpha}\right)= \begin{cases}A(\phi ; \alpha, s, p) z^{\alpha+s-p} & \alpha+s \geq p, \\
B(\phi ; p, \alpha, s) \bar{z}^{p-\alpha-s} & \alpha+s \leq p, \\
0 & \text { others. }\end{cases}
\end{aligned}
$$

Note that, for two nonzero multi-indexes $p, s \in \mathbb{N}^{n}$ with $p \perp s$, then $\alpha+p \geq s$ if and only if $\alpha \geq s$ and there is no $\alpha \in \mathbb{N}^{n}$ such that $\alpha+p \leq s$. Hence the above lemma gives the following.

Lemma 8. Let $p, s \in \mathbb{N}^{n}$ with $p, s \neq 0$ and $p \perp s, \phi \in \mathscr{R}$; then, for any multi-index $\alpha \in \mathbb{N}^{n}$,

$$
\begin{aligned}
& H_{\xi^{p} \bar{\xi}_{\phi}^{s}}\left(z^{\alpha}\right)= \begin{cases}A(\phi ; \alpha, p, s) \bar{z}^{\alpha+p-s} & \alpha \geq s, \\
0 & \text { others, }\end{cases} \\
& H_{\xi^{p} \bar{\xi}^{s} \phi}\left(\bar{z}^{\alpha}\right)= \begin{cases}A(\phi ; \alpha, s, p) z^{\alpha+s-p} & \alpha \geq p, \\
0 & \text { others. }\end{cases}
\end{aligned}
$$

We are ready to prove the first main result.

Proof of Theorem 1. Using Lemma 7, direct calculations show that

$$
\begin{aligned}
& H_{\xi p \bar{\xi}^{s} \phi} H_{\xi^{q} \bar{\xi}^{t} \psi}\left(z^{\alpha}\right) \\
& \quad=A(\psi ; \alpha, q, t) A(\phi ; \alpha+q-t, s, p) z^{\alpha+q-t+s-p}
\end{aligned}
$$

when $\alpha \geq t-q, \alpha \geq t-q+p-s$ and

$$
\begin{aligned}
& H_{\xi^{q} \bar{\xi}^{t} \psi} H_{\xi p \bar{\xi}^{s} \phi}\left(z^{\alpha}\right) \\
& \quad=A(\phi ; \alpha, p, s) A(\psi ; \alpha+p-s, t, q) z^{\alpha+p-s+t-q}
\end{aligned}
$$

when $\alpha \geq s-p, \alpha \geq s-p+q-t$. Thus when

$$
\alpha \geq t-q, t-q+p-s, s-p, q-t+s-p,
$$

combining with (21) and (22), it follows from $\left[H_{\xi p} \bar{\xi}^{s} \phi^{\prime}\right.$ $\left.H_{\xi^{q} \bar{\xi}_{\psi}^{t}}\right]\left(z^{\alpha}\right)=0$ that

$$
\alpha+q-t+s-p=\alpha+p-s+t-q
$$

which implies $p-s=q-t$ or

$$
A(\psi ; \alpha, q, t) A(\phi ; \alpha+q-t, s, p)=0
$$

holds when $\alpha \geq t-q, \alpha \geq t-q+p-s$, which induces $\phi=0$ or $\psi=0$. In fact, for the sake of simplicity, we only consider the case $n=2$. Put

$$
E=\left\{\alpha \in \mathbb{N}^{2}: \alpha \geq p+s, A(\psi ; \alpha, q, t)=0\right\},
$$


if $E$ satisfies the condition (I); then, by Lemma 6, we can get $\psi=0$; otherwise $E^{c}$ will satisfy the condition (I), which by (25) says that the set

$$
\left\{\alpha \in \mathbb{N}^{2}: \alpha \geq p+s, A(\phi ; \alpha+q-t, s, p)=0\right\}
$$

satisfies the condition (I); thus, by Lemma 6 , again we get $\phi=$ 0 .

Conversely, if $\phi=0$ or $\psi=0$, it is clear that $H_{\xi^{p} \bar{\xi}^{s} \phi}$ commutes with $H_{\xi^{q} \bar{\xi}^{t} \psi}$. Now suppose $p-s=q-t$. When $\alpha \geq t-q$ and $\alpha \geq 0$, by (21) and (22), one can easily check

$$
H_{\xi^{p} \bar{\xi}_{\phi}^{s}} H_{\xi^{q} \bar{\xi}^{t} \psi}\left(z^{\alpha}\right)=H_{\xi^{q} \bar{\xi}^{t} \psi} H_{\xi^{p} \bar{\xi}_{\phi}^{s}}\left(z^{\alpha}\right)
$$

since $p-s=q-t$. When $0 \leq \alpha \leq t-q$, by Lemma 7 , we have

$$
\begin{aligned}
& H_{\xi^{p} \bar{\xi}_{\phi}^{s}} H_{\xi^{q} \bar{\xi}^{t} \psi}\left(z^{\alpha}\right) \\
& \quad=B(\psi ; t, \alpha, q) B(\phi ; s, t-\alpha-q, p) z^{\alpha}, \\
& H_{\xi^{q} \bar{\xi}^{t} \psi} H_{\xi^{p} \bar{\xi}^{s} \phi}\left(z^{\alpha}\right) \\
& \quad=B(\phi ; s, \alpha, p) B(\psi ; t, s-\alpha-p, q) z^{\alpha} .
\end{aligned}
$$

It is clear that the above two are equal because $p-s=q-t$. When $\alpha \geq 0$ and $t-q \not \alpha \not t-q$, both sides of equality (28) are zero, so they are equal. Thus, we conclude $H_{\xi p} \bar{\xi}^{s} H_{\xi^{q} \bar{\xi}^{t} \psi}=$ $H_{\xi^{q} \bar{\xi}^{t} \psi} H_{\xi^{p} \bar{\xi}^{s} \phi}$ on the analytic part $\left\{z^{\alpha}\right\}_{\alpha \in \mathbb{N}^{n}}$, which is also true on the coanalytic part $\left\{\bar{z}^{\alpha}\right\}_{\alpha \in \mathbb{N}^{n}}$ with similar arguments when $p-s=q-t$. The proof is complete.

Before we prove Theorem 2, we introduce the Mellin transform which is one of the most useful tools in studying our problems. The Mellin transform $\hat{\phi}$ of a function $\phi \epsilon$ $L^{1}([0,1], r d r)$ is defined by $\widehat{\phi}(z)=\int_{0}^{1} \phi(s) s^{z-1} d s$. It is known that $\widehat{\phi}$ is a bounded analytic function in the half plane $\{z$ : $\operatorname{Re} z>2\}$. In addition, for a radial function $\phi$, to compute $\int_{\mathbb{B}_{n}} \phi(z)\left|z^{\alpha}\right|^{2} d v$ using (13), we obtain

$$
A(\phi ; \alpha, 0,0)=(2 n+2|\alpha|) \widehat{\phi}(2 n+2|\alpha|) .
$$

Let $z=\left(z_{1}, \ldots, z_{n}\right)=\left(|z| \xi_{1}, \ldots,|z| \xi_{n}\right) \in \mathbb{B}_{n}$, where $\xi=$ $\left(\xi_{1}, \ldots, \xi_{n}\right) \in \mathbb{S}_{n}$. Put $r_{j}=\left|z_{j}\right|$, then $\left|\xi_{j}\right|=r_{j} /|z|, j=1, \ldots, n$. Let $r=\left(r_{1}, \ldots, r_{n}\right)$. For every separately quasihomogeneous function $f(z)=f(|r| \xi)=\xi^{\beta} \bar{\xi}^{\gamma} \phi(r)$, there is unique $p, s \in \mathbb{N}^{n}$ with $p \perp s$ such that $p-s=\beta-\gamma$ and

$$
\begin{aligned}
& f(|r| \xi)=\xi^{p} \bar{\xi}^{s}\left|\xi_{1}\right|^{2 \min \left\{\beta_{1}, \gamma_{1}\right\}} \cdots\left|\xi_{n}\right|^{2 \min \left\{\beta_{n}, \gamma_{n}\right\}} \phi(r) \\
& =\xi^{p} \bar{\xi}^{s}\left(\frac{r_{1}}{|r|}\right)^{2 \min \left\{\beta_{1}, \gamma_{1}\right\}} \cdots\left(\frac{r_{n}}{|r|}\right)^{2 \min \left\{\beta_{n}, \gamma_{n}\right\}} \phi(r) \\
& =: \xi^{p} \bar{\xi}^{s} \Phi(r),
\end{aligned}
$$

where $\Phi$ is a separately radial function. Thus for each $h \epsilon$ $L^{2}\left(\mathbb{B}_{n}, d v\right)$ denoted as the form (14), we may rewrite $h=h_{1}+$ $h_{2}+h_{3}$, where

$$
\begin{aligned}
& h_{1}=\sum_{p \geq 0} \xi^{p} \phi_{p, 0}, \\
& h_{2}=\sum_{s \geq 0, s \neq 0} \bar{\xi}^{s} \phi_{0, s}, \\
& h_{3}=\sum_{p \perp s, p, s \neq 0} \xi^{p} \bar{\xi}^{s} \phi_{p, s} .
\end{aligned}
$$

Now we can prove the second main result.

Proof of Theorem 2. Suppose $\left[H_{\phi}, H_{h}\right]=0$. We may write $h=$ $h_{1}+h_{2}+h_{3}$, where $h_{1}, h_{2}, h_{3}$ are given by (32). Using Lemmas 7 and 8 , direct calculations give that, for every $\alpha \in \mathbb{N}^{n}$,

$$
\begin{aligned}
& H_{\phi} H_{h}\left(z^{\alpha}\right)=H_{\phi} H_{h_{1}}\left(z^{\alpha}\right)+H_{\phi} H_{h_{2}}\left(z^{\alpha}\right) \\
& +H_{\phi} H_{h_{3}}\left(z^{\alpha}\right)=\sum_{p \geq 0} A\left(\phi_{p, 0} ; \alpha, p, 0\right) \\
& \cdot A(\phi ; \alpha+p, 0,0) z^{\alpha+p}+\sum_{s \geq \alpha, s \neq \alpha} B\left(\phi_{0, s} ; s, \alpha, 0\right) \\
& \cdot A(\phi ; s-\alpha, 0,0) \bar{z}^{s-\alpha}+\sum_{0 \neq s \leq \alpha} A\left(\phi_{0, s} ; \alpha, s, 0\right) \\
& \cdot A(\phi ; \alpha-s, 0,0) z^{\alpha-s}+\sum_{p \perp s, p, s \neq 0, s \leq \alpha} A\left(\phi_{p, s} ; \alpha, p, s\right) \\
& \cdot A(\phi ; \alpha+p-s, 0,0) z^{\alpha+p-s}, \\
& H_{h} H_{\phi}\left(z^{\alpha}\right)=A(\phi ; \alpha, 0,0) H_{h}\left(\bar{z}^{\alpha}\right) \\
& =\sum_{0 \neq p \leq \alpha} A\left(\phi_{p, 0} ; \alpha, 0, p\right) A(\phi ; \alpha, 0,0) z^{\alpha-p} \\
& +\sum_{p \geq \alpha, p \neq \alpha} B\left(\phi_{p, 0} ; p, \alpha, 0\right) A(\phi ; \alpha, 0,0) \bar{z}^{p-\alpha} \\
& +\sum_{s \geq 0} A\left(\phi_{0, s} ; \alpha, s, 0\right) A(\phi ; \alpha, 0,0) z^{\alpha+s} \\
& +\sum_{p \perp s, p, s \neq 0, p \leq \alpha} A\left(\phi_{p, s} ; \alpha, s, p\right) A(\phi ; \alpha, 0,0) z^{\alpha+s-p} .
\end{aligned}
$$

Comparing the fourth summations in the equalities (33) and (34), it follows from $H_{\phi} H_{h}\left(z^{\alpha}\right)=H_{h} H_{\phi}\left(z^{\alpha}\right)$ that, for every $p \perp s, p, s \neq 0$ and $s \leq \alpha$, we have

$$
\begin{gathered}
A\left(\phi_{p, s} ; \alpha, p, s\right) A(\phi ; \alpha+p-s ; 0,0) \\
\quad=A\left(\phi_{s, p} ; \alpha, p, s\right) A(\phi ; \alpha, 0,0) .
\end{gathered}
$$

Similarly, comparing the first summation in the equality (33) with the third one in the equality (34), we then get that, for every $p, \alpha \geq 0$,

$$
\begin{aligned}
& A\left(\phi_{p, 0} ; \alpha, p, 0\right) A(\phi ; \alpha+p ; 0,0) \\
& \quad=A\left(\phi_{0, p} ; \alpha, p, 0\right) A(\phi ; \alpha, 0,0) .
\end{aligned}
$$


With the same arguments as above, it follows from $H_{\phi} H_{h}\left(\bar{z}^{\alpha}\right)=H_{h} H_{\phi}\left(\bar{z}^{\alpha}\right)$ that, for every $p \perp s, p, s \neq 0$ and $s \leq \alpha$, we have

$$
\begin{gathered}
A\left(\phi_{s, p} ; \alpha, p, s\right) A(\phi ; \alpha+p-s ; 0,0) \\
\quad=A\left(\phi_{p, s} ; \alpha, p, s\right) A(\phi ; \alpha, 0,0)
\end{gathered}
$$

and, for every $p, \alpha \geq 0$,

$$
\begin{aligned}
A & \left(\phi_{0, p} ; \alpha, p, 0\right) A(\phi ; \alpha+p ; 0,0) \\
& =A\left(\phi_{p, 0} ; \alpha, p, 0\right) A(\phi ; \alpha, 0,0) .
\end{aligned}
$$

Suppose $\phi$ is a nonzero constant; then, by (30), we have

$$
A(\phi ; \alpha+p-s ; 0,0)=A(\phi ; \alpha, 0,0) \neq 0,
$$

so (35) gives that, for every $p \perp s, p, s \neq 0$, when $\alpha \geq s$,

$$
A\left(\phi_{p, s}-\phi_{s, p} ; \alpha, p, s\right)=0
$$

which induces that $\phi_{p, s}=\phi_{s, p}$ for every $p \perp s, p, s \neq 0$ by Lemma 6. Similarly, it follows from (36) we can get $\phi_{p, 0}=\phi_{0, p}$ for every $p \neq 0$. Therefore, we obtain $J h=h$.

In the following, we suppose $\phi$ is not a constant. It follows from (35) and (37) that, for every $p \perp s, p, s \neq 0$, when $\alpha \geq s$,

$$
\begin{aligned}
& A\left(\phi_{p, s}-\phi_{s, p} ; \alpha, p, s\right) \\
& \quad \cdot[A(\phi ; \alpha+p-s, 0,0)+A(\phi ; \alpha, 0,0)]=0 .
\end{aligned}
$$

For the sake of simplicity, we only consider the case $n=2$. For $p \perp s, p, s \neq 0$, put

$$
E_{p, s}=\left\{\alpha \in \mathbb{N}^{n}: \alpha \geq s, A\left(\phi_{p, s}-\phi_{s, p} ; \alpha, p, s\right)=0\right\} .
$$

If $E_{p, s}$ satisfies the condition (I), then by Lemma 6 we obtain $\phi_{p, s}=\phi_{s, p}$; if $E_{p, s}$ does not satisfy the condition (I), then $E_{p, s}^{c}$, the complement of $E_{p, s}$ in $\left\{\alpha \in \mathbb{N}^{n}: \alpha \geq s\right\}$, must satisfy the condition (I). In particular, we have $\sum_{\alpha \in E_{p, s}^{c}}(1 /|\alpha|)=\infty$. So by (41) we have, for $p \perp s, p, s \neq 0$,

$$
A(\phi ; \alpha, 0,0)=-A(\phi ; \alpha+p-s, 0,0), \quad \alpha \in E_{p, s}^{c} .
$$

That is, when $\alpha \in E_{p, s}^{c}$, using (30), we have

$$
\begin{aligned}
(2 n+2|\alpha|) \widehat{\phi}(2 n+2|\alpha|) & \\
= & -(2 n+2|\alpha|+2|p|-2|s|) \\
\cdot & \widehat{\phi}(2 n+2|\alpha|+2|p|-2|s|) .
\end{aligned}
$$

Denote

$$
\begin{aligned}
F(z)= & \widehat{\phi}(z+2 n+2|p|-2|s|) \\
& +\frac{z+2 n}{z+2 n+2|p|-2|s|} \widehat{\phi}(z+2 n),
\end{aligned}
$$

and then $F$ is a bounded analytic function on $\{z \in \mathbb{C}: \operatorname{Re} z>$ $2|s|\}$. Moreover, it follows from (44) that $F(2|\alpha|)=0$ when $\alpha \in E_{p, s}^{c}$. According to Lemma 5 we thus have $F \equiv 0$, which implies

$$
\begin{aligned}
& (z+2 n) \widehat{\phi}(z+2 n) \\
& \quad=-(z+2 n+2|p|-2|s|) \widehat{\phi}(z+2 n+2|p|-2|s|)
\end{aligned}
$$

for every $z$ in $\{z \in \mathbb{C}: \operatorname{Re} z>2|s|\}$. If $|p|=|s|$, then it follows from the above that $2(z+2 n) \widehat{\phi}(z+2 n)=0$, so it is clear that $\phi=0$, a contradiction. Without loss of generality, we may assume $|p|>|s|$. Replace $z$ by $z+2|p|-2|s|$ in (46) and then compare with (46) to obtain

$$
\begin{aligned}
(z & +2 n) \hat{\phi}(z+2 n) \\
& =(z+2 n+4|p|-4|s|) \widehat{\phi}(z+2 n+4|p|-4|s|),
\end{aligned}
$$

and thus, as in the proof of Theorem 6 in [6], the above equation gives that $\phi$ is a constant, which is also a contradiction.

Therefore, when $\phi$ is not a constant, then, for every $p, s \in$ $\mathbb{N}^{n}$ with $p \perp s$ and $p, s \neq 0, \phi_{p, s}=\phi_{s, p}$. Putting them into (35), we get that, for $p \perp s, p, s \neq 0$,

$$
\begin{aligned}
& A\left(\phi_{p, s} ; \alpha, p, s\right)[A(\phi ; \alpha+p-s ; 0,0)-A(\phi ; \alpha, 0,0)] \\
& \quad=0
\end{aligned}
$$

when $\alpha \geq s$. Since $\phi$ is not a constant, then similar argument as before we get $\phi_{p, s}=0$; hence $\phi_{p, s}=\phi_{s, p}=0$ for $p \perp$ $s, p, s \neq 0$. With same arguments, using (36) and (38), we can get $\phi_{p, 0}=\phi_{0, p}=0$ for $p \neq 0$. So $h=\phi_{0,0}$, a separately radial function.

Conversely, if $\phi=0$, then clearly $\left[H_{\phi}, H_{h}\right]=0$; if $h$ is separately radial, then Theorem 1 gives $\left[H_{\phi}, H_{h}\right]=0$; if $\phi=c$, a nonzero constant, and $J h=h$, then using (9) we have

$$
H_{c} H_{h}=c J H_{h}=c H_{J h} J=H_{J h} H_{c}=H_{h} H_{c},
$$

as desired. The proof is complete.

Before we prove the last two main results, we should note that, for every small Hankel operator $H_{h}$ with $h \in L^{\infty}$, it is easy to check that $H_{h}$ is a complex symmetric operator with complex conjugate $C$ which is defined as $C f(z)=\overline{f(\bar{z})}$ for $f \in$ $L^{2}\left(\mathbb{B}_{n}, d v\right)$, that is, $H_{f}^{*}=C H_{h} C$. Hence we have the following analogous result as Proposition 18 in [14].

Lemma 9. Let $f, g, h \in L^{\infty}$. Then $H_{f} H_{g}=H_{h}$ if and only if $H_{g} H_{f}=H_{h}$, and each case implies $\left[H_{f}, H_{g}\right]=0$.

We also need the following partial result of zero product characterization which has independent interest.

Theorem 10. Let $p, s \in \mathbb{N}^{n}, \phi \in \mathscr{R} \cap L^{\infty}, h \in L^{\infty}$. Then the following statements are equivalent:

(i) $H_{\xi^{p} \bar{\xi}_{\phi}^{s}} H_{h}=0$.

(ii) $H_{h} H_{\xi^{p} \bar{\xi}_{\phi}^{s}}=0$.

(iii) $\phi=0$ or $h=0$. 
Proof. By Lemma 9 we only need to show (ii) $\Rightarrow$ (iii). Write $h$ as the form (14). When $\alpha \geq s-p$, by Lemma 7, we have

$$
H_{\xi p \bar{\xi}^{s} \phi}\left(z^{\alpha}\right)=A(\phi ; \alpha, p, s) \bar{z}^{\alpha+p-s} .
$$

Using Lemma 7 again we get that the analytic part of $H_{h} H_{\xi p \bar{\xi}_{\phi}^{s}}\left(z^{\alpha}\right)$ is given by

$$
\begin{aligned}
& \sum_{\beta-\gamma+p-s \leq \alpha} A(\phi ; \alpha, p, s) A\left(\phi_{\beta, \gamma} ; \alpha+p-s, \gamma, \beta\right) \\
& \cdot z^{\alpha+p-s+\gamma-\beta}
\end{aligned}
$$

and hence $H_{h} H_{\xi p} \bar{\xi}_{\phi}^{s}\left(z^{\alpha}\right)=0$ will give that, for each $\beta, \gamma \in \mathbb{N}^{n}$,

$$
A(\phi ; \alpha, p, s) A\left(\phi_{\beta, \gamma} ; \alpha+p-s, \gamma, \beta\right)=0
$$

holds for any $\alpha \geq s-p$ and $\alpha \geq \beta-\gamma+p-s$. Therefore, with the same arguments as done in the proof of Theorem 1 we can get $\phi=0$ or $\phi_{\beta, \gamma}=0$ for each $\beta, \gamma$, as the assertion. We finish the proof.

The next rephrasing of above result is a cancellation law for small Hankel operators with separately quasihomogeneous symbols.

Corollary 11. Let $p, s \in \mathbb{N}^{n}$ and $0 \neq \phi \in \mathscr{R} \cap L^{\infty}, g, h \in L^{2}$. Then the following statements are equivalent:

(i) $H_{\xi^{p} \bar{\xi} \phi}^{s} H_{g}=H_{\xi^{p} \bar{\xi}_{\phi}^{s}} H_{h}$.

(ii) $H_{g} H_{\xi^{p} \bar{\xi}_{\phi}^{s}}=H_{h} H_{\xi^{p} \bar{\xi}_{\phi}^{s}}$.

(iii) $g=h$.

Now we turn to prove the third main result.

Proof of Theorem 3. By Lemma 9 we only need to show (i) $\Rightarrow$ (iii). Write $h$ as the form (14). Using Lemma 7, direct calculations give that (21) holds when $\alpha \geq t-q, \alpha \geq t-q+p-s$ and

$$
\begin{aligned}
H_{h}\left(z^{\alpha}\right)= & \sum_{\gamma-\beta \leq \alpha} A\left(\phi_{\beta, \gamma} ; \alpha, \beta, \gamma\right) \bar{z}^{\alpha+\beta-\gamma} \\
& +\sum_{\gamma-\beta \geq \alpha, \gamma-\beta \neq \alpha} B\left(\phi_{\beta, \gamma} ; \gamma, \alpha, \beta\right) z^{\gamma-\alpha-\beta},
\end{aligned}
$$

and hence when $\alpha \geq t-q, \alpha \geq t-q+p-s$, the two equalities (21) and (53) are equal implying that the coanalytic part of $H_{h}\left(z^{\alpha}\right)$ must be zero, that is, $A\left(\phi_{\beta, \gamma} ; \alpha, \beta, \gamma\right)=0$ for all $\alpha \geq$ $\gamma-\beta, \alpha \geq t-q, \alpha \geq t-q+p-s$.

Now for each fixed $\beta, \gamma \in \mathbb{N}^{n}$, using Lemma 6 , the above tells $\phi_{\beta, \gamma}=0$, which yields that $h=0$. Hence we get

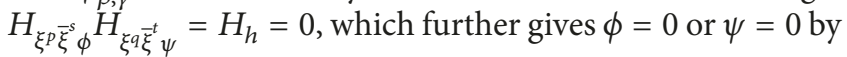
Theorem 10. We complete the proof.

Theorem 3 answers immediately the following semicommutativity for two small Hankel operators with separately radial symbols.
Corollary 12. Let $p, s, q, t \in \mathbb{N}^{n}$ and $\phi, \psi \in \mathscr{R} \cap L^{\infty}$. Then the following statements are equivalent:

(i) $H_{\xi^{p} \bar{\xi}_{\phi}^{s}} H_{\xi^{q} \bar{\xi}_{\psi}^{t} \psi}=H_{\xi^{p+q} \bar{\xi}_{\phi \psi}^{s+t}}$.

(ii) $H_{\xi^{q} \bar{\xi}^{t} \psi} H_{\xi^{p} \bar{\xi}_{\phi}^{s}}=H_{\xi^{p+q} \bar{\xi}^{s+t} \phi \psi}$.

(iii) $\phi=0$ or $\psi=0$.

The next corollary says there are no nonzero idempotent small Hankel operators with separately quasihomogeneous symbols.

Corollary 13. Let $p, s \in \mathbb{N}^{n}$ and $\phi \in \mathscr{R} \cap L^{\infty}$. If $H_{\xi^{p} \bar{\xi}_{\phi}^{s}}^{2}=$ $H_{\xi^{p} \bar{\xi}^{s} \phi}$ then $\phi=0$.

Now it is left to prove the last main result. We need the following lemma which is a generalization of Lemma 19 in [14] to higher dimension case.

Lemma 14. Let $g, h \in L^{\infty}$. Then $T_{h}=H_{g}$ if and only if $g=$ $h=0$.

Proof. Put $\widetilde{T}_{h}:=P T_{h} P$ and $\widetilde{H}_{g}:=P H_{g} P$; then $\widetilde{T}_{h}$ and $\widetilde{H}_{g}$ are Toeplitz operator and small Hankel operator on the Bergman space $L_{a}^{2}\left(\mathbb{B}_{n}\right)$, respectively. It is easy to verify that

$$
\widetilde{T}_{\overline{z_{j}}} \widetilde{H}_{h}=\widetilde{H}_{h} \widetilde{T}_{z_{j}}, \quad j=1,2, \ldots, n .
$$

Suppose $T_{h}=H_{g}$; then $\widetilde{T}_{g}=\widetilde{H}_{h}$. Applying the above equations we get

$$
\widetilde{T}_{\overline{z_{j}} g}=\widetilde{T}_{\overline{z_{j}}} \widetilde{T}_{g}=\widetilde{T}_{\overline{z_{j}}} \widetilde{H}_{h}=\widetilde{H}_{h} \widetilde{T}_{z_{j}}=\widetilde{T}_{g} \widetilde{T}_{z_{j}}=\widetilde{T}_{z_{j} g}
$$

to obtain $\widetilde{T}_{\overline{z_{j}} g-z_{j} g}=0$, which gives $\left(\overline{z_{j}}-z_{j}\right) g=0$, and so $g=0$. It follows from $H_{h}=T_{g}=0$ that $h=0$. The proof is complete.

Proof of Theorem 4. Supposing $H_{\phi} H_{h}=H_{g}$, then by Lemma 9 we get $\left[H_{\phi}, H_{h}\right]=0$. So Theorem 2 tells that one of the following cases may occur.

Case (a). $(\phi=0$.) In this case, we get $\phi=g=0$.

Case (b). ( $h$ is a separately radial function.) In this case, by Theorem 3, we have $\phi=g=0$ or $h=g=0$.

Case $(c) .(\phi$ is a nonzero constant and $J h=h$.) Without loss of generality, we assume $\phi=1$. Combining with (9) we have $H_{g}=H_{1} H_{h}=J H_{h}=T_{h}$. Hence by Lemma 14 we get $g=h=$ 0 . proof.

The remaining of the proof is clear. We complete the

The following semicommutativity is obtained easily by Theorem 4.

Corollary 15. Let $\phi$ be bounded radial and $g \in L^{\infty}$. Then the following statements are equivalent: 
(i) $H_{\phi} H_{g}=H_{\phi g}$.

(ii) $H_{g} H_{\phi}=H_{\phi g}$.

(iii) $\phi=0$ or $g=0$.

\section{Conflicts of Interest}

The authors declare that they have no conflicts of interest.

\section{Acknowledgments}

The first three authors were supported by NSFC (nos. 11771401 and 11471113) and ZJNSFC (no. LY14A010013). The last author was supported by ZJNSFC (no. LY13A010021).

\section{References}

[1] A. Brown and P. R. Halmos, "Algebraic properties of Toeplitz operators," Journal für die reine und Angewandte Mathematik, vol. 213, pp. 89-102, 1964.

[2] R. Martinez-Avendano, "When do Toeplitz and Hankel operators commute?" Integral Equations and Operator Theory, vol. 37, no. 3, pp. 341-349, 2000.

[3] K. Guo and D. Zheng, "Essentially commuting Hankel and Toeplitz operators," Journal of Functional Analysis, vol. 201, no. 1, pp. 121-147, 2003.

[4] P. Ahern and P. Z. Cuckovic, "A theorem of Brown-Halmos type for BERgman space Toeplitz operators," Journal of Functional Analysis, vol. 187, no. 1, pp. 200-210, 2001.

[5] S. Axler and Z. Cuckovic, "Commuting Toeplitz operators with harmonic symbols," Integral Equations and Operator Theory, vol. 14, no. 1, pp. 1-12, 1991.

[6] Z. Cuckovic and N. V. Rao, "Mellin transform, monomial symbols, and commuting Toeplitz operators," Journal of Functional Analysis, vol. 154, no. 1, pp. 195-214, 1998.

[7] I. Louhichi, E. Strouse, and L. Zakariasy, "Products of Toeplitz operators on the Bergman space," Integral Equations and Operator Theory, vol. 54, no. 4, pp. 525-539, 2006.

[8] I. Louhichi and L. Zakariasy, "On Toeplitz operators with quasihomogeneous symbols," Archiv der Mathematik, vol. 85, no. 3, pp. 248-257, 2005.

[9] Y. F. Lu and B. Zhang, "Commuting Hankel and Toeplitz operators on the Bergman space," Chinese Annals of Mathematics. Series A. Shuxue Niankan. A Ji, vol. 32, no. 5, pp. 519-530, 2011.

[10] B. R. Choe and Y. J. Lee, "Commuting Toeplitz operators on the harmonic Bergman space," Michigan Mathematical Journal, vol. 46, no. 1, pp. 163-174, 1999.

[11] X. Ding, "A question of Toeplitz operators on the harmonic Bergman space," Journal of Mathematical Analysis and Applications, vol. 344, no. 1, pp. 367-372, 2008.

[12] X.-T. Dong and Z.-H. Zhou, "Commuting quasihomogeneous Toeplitz operators on the harmonic Bergman space," Complex Analysis and Operator Theory, vol. 7, no. 4, pp. 1267-1285, 2013.

[13] X.-T. Dong and Z.-H. Zhou, "Products of Toeplitz operators on the harmonic Bergman space," Proceedings of the American Mathematical Society, vol. 138, no. 5, pp. 1765-1773, 2010.

[14] Y. Chen, W. He, and Y. Hu, "Algebraic properties of small Hankel operators on the harmonic Bergman space," Illinois Journal of Mathematics, vol. 59, no. 2, pp. 295-317, 2015.
[15] X.-T. Dong and Z.-H. Zhou, "Algebraic properties of Toeplitz operators with separately quasihomogeneous symbols on the Bergman space of the unit ball," The Journal of Operator Theory, vol. 66, no. 1, pp. 193-207, 2011.

[16] R. Remmert, Classical Topics in Complex Function Theory, Graduate Texts in Mathematics, Springer, New York, NY, USA, 1998.

[17] H. Guan, L. Liu, and Y. Lu, "Algebraic properties of quasihomogeneous and separately quasihomogeneous toeplitz operators on the pluriharmonic bergman space," Abstract and Applied Analysis, vol. 2013, Article ID 252037, 12 pages, 2013. 


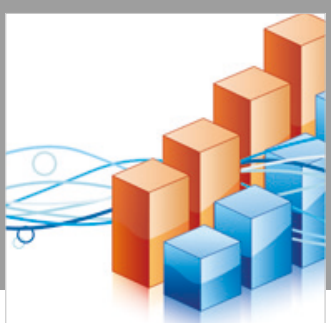

Advances in

Operations Research

\section{-n-m}
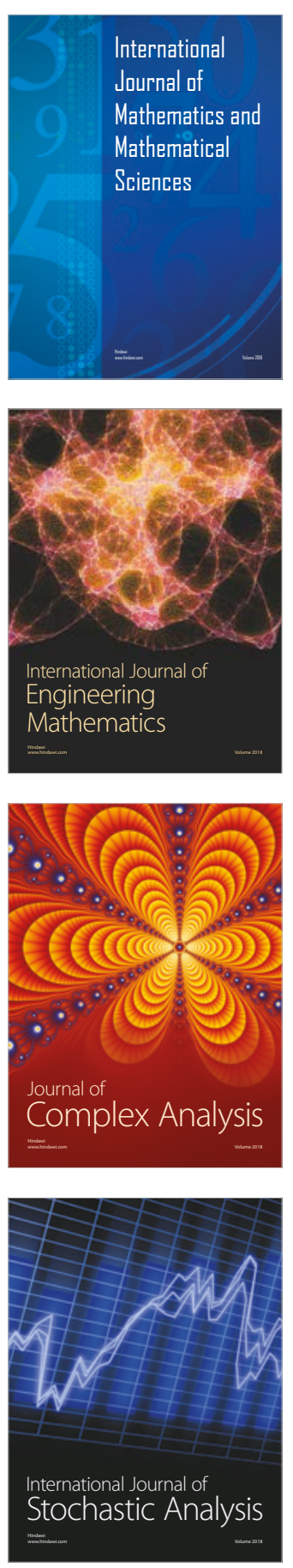
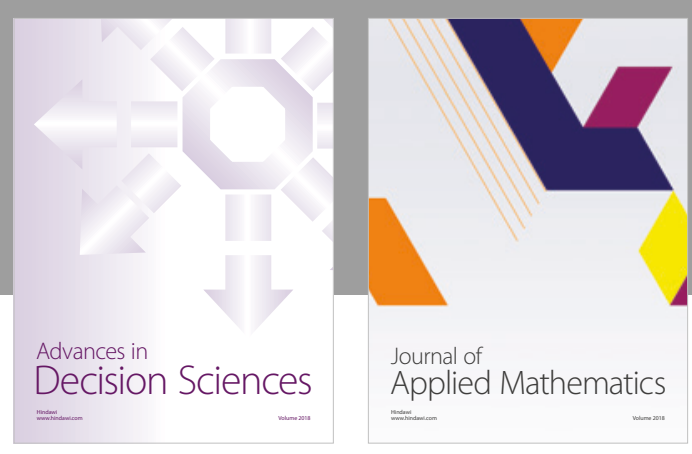

Journal of

Applied Mathematics
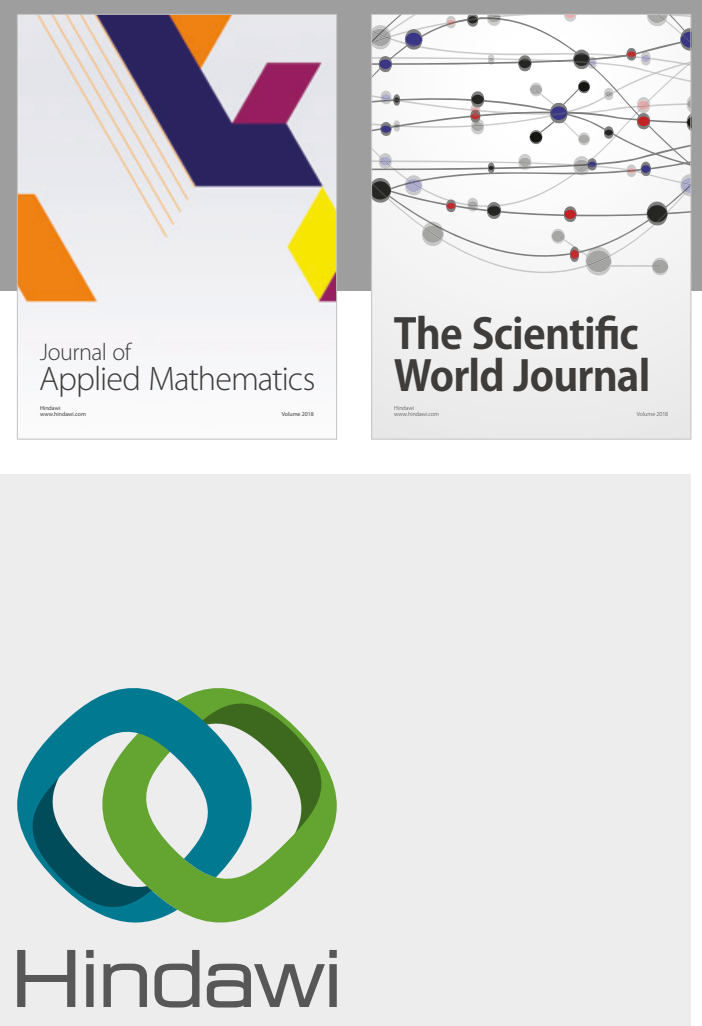

Submit your manuscripts at

www.hindawi.com

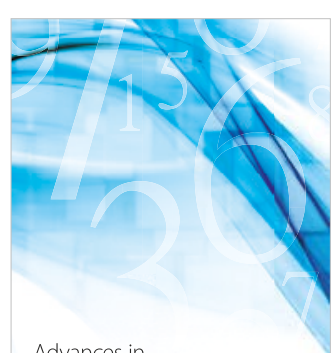

Advances in
Numerical Analysis
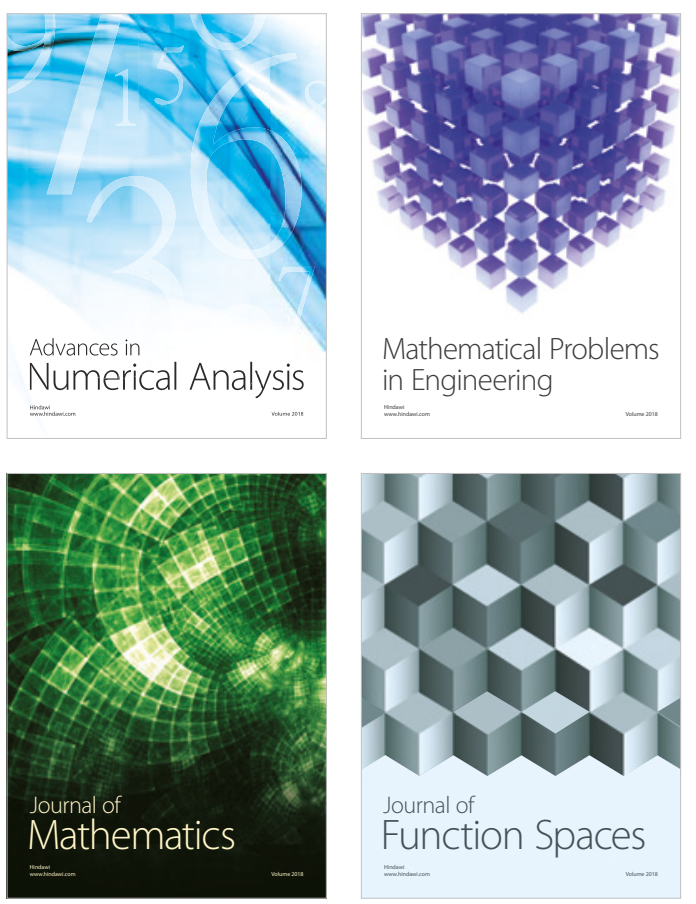

Mathematical Problems in Engineering

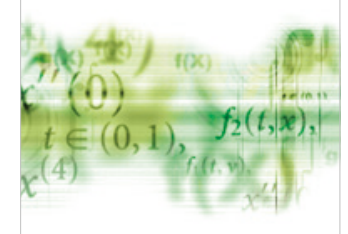

International Journal of

Differential Equations

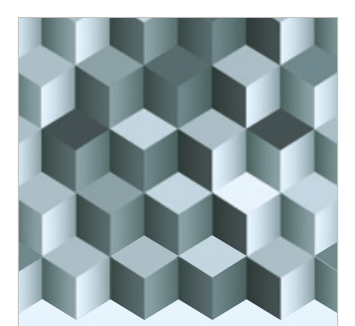

Journal of

Function Spaces

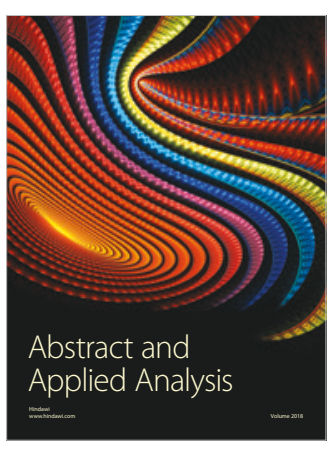

The Scientific

World Journal

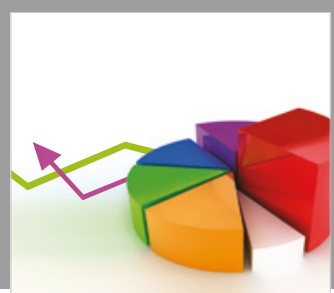

Journal of

Probability and Statistics
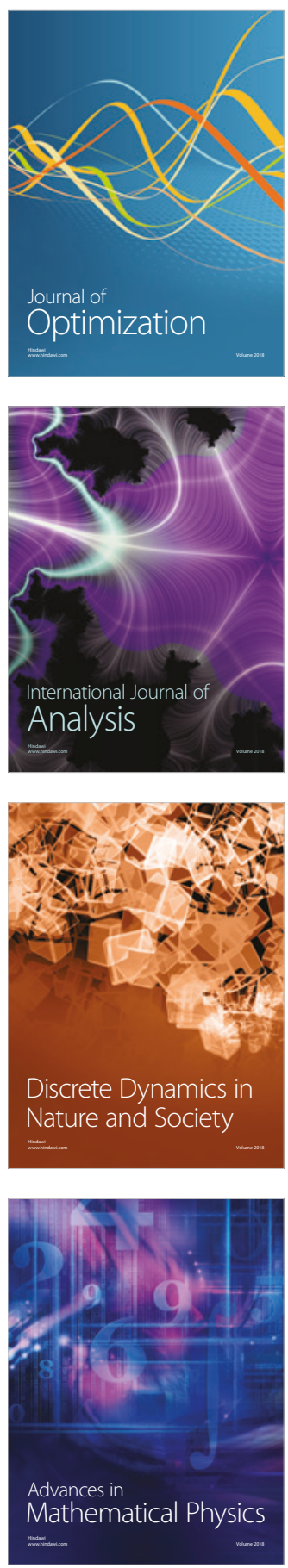